\title{
Application of Neutrosophic Similarity Measures in Covid-19
}

\author{
Rakhal Das $^{1} \cdot$ Anjan Mukherjee $^{1} \cdot$ Binod Chandra Tripathy $^{1}(\mathbb{D}$ \\ Received: 11 March 2021 / Revised: 18 October 2021 / Accepted: 8 November 2021 / \\ Published online: 2 December 2021 \\ ๑) The Author(s), under exclusive licence to Springer-Verlag GmbH Germany, part of Springer Nature 2021
}

\begin{abstract}
The contemporary situation of the world is very pathetic due to the spread of COVID19. In this article, we have prepared a decision making model on COVID-19 pandemic patients with the help of the neutrosophic similarity measures. The model is to predict the COVID-19 patents for testing positive and testing negative. The decision making is based on the testing result of the COVID-19 cases. We have used the neutrosophic similarity measure theory and the distance function. We have used the C-programming for finding the result of the suspected patients.
\end{abstract}

Keywords Neutrosophic set · Raw data - Corona virus · Similarity measure ·

Distance function C-programming

Mathematical Subject Classification 03E72 $\cdot 90 \mathrm{~B} 50 \cdot 03 \mathrm{E} 75$

\section{Introduction}

The World Health Organization (WHO) announced a pandemic crisis due to COVID19 on March 11, 2020. COVID-19 has a variety of effects on various persons. The COVID disease manifests itself in six different ways. The majority of infected patients will experience mild to moderate sickness and will be able to recover without the need for hospitalization.

COVID-19 was discovered for the first time in December 2019 in Wuhan, China. On January 30, 2020, the World Health Organization designated the outbreak a Public

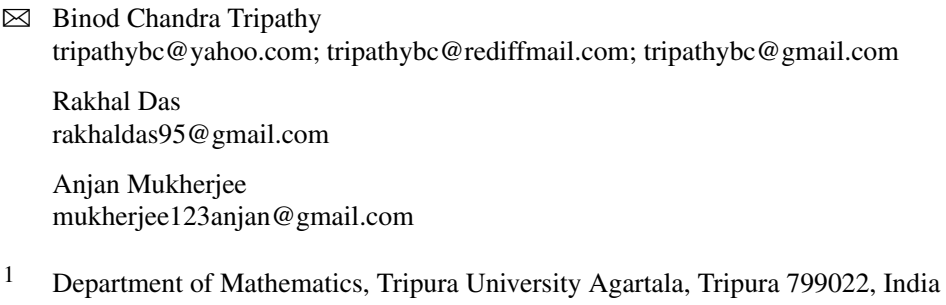


Health Emergency of International Concern, and on March 11, it was declared a pandemic. More than 199,022,838 cases had been documented in more than 222 nations and territories as of August 2, 2021, resulting in more than 4,240,374 deaths; more than $179,631,883$ people have recovered. There are many symptoms of COVID19 pandemic patents. In this article, we have developed a model for the decision on becoming positive or negative of the COVID patents. As a result of which the patients will be admitted for treatment to save life. We have taken the help of the neutrosophic set theory for the case study, since the number of symptomatic people is asymptomatic. After the analysis of the tested data, one can decide the necessity for ventilation and who are home-quarantine process. We use the similarity measure theory for the distance measure of the COVID-19 patents. The terms "Similarity" is an adjective, the term may refer to a likeness, analogy, semblance, or comparableness between several aspects, incidents, objects, or even beings. Two objects are said to be similar if they are alike to a certain degree in terms of their inherent attributes such as appearance (viz. size, texture, color, height, length, broadness, thickness, etc.), nature or behavior, or in terms of the way they react or interact concerning other objects or beings. In mathematics and the related fields, the logical counterpart of similarity exists as a real-valued function that estimates the similitude between objects. The concept of Intuitionistic fuzzy sets, interval valued Intuitionistic fuzzy sets and soft set was studied by many researchers [1-6]. The concept of similarity in the context of uncertainty (in the form of fuzziness) can be traced back to the study of similarity measure introduced by [7-16], proposed an axiomatic definition of similarity. Xuecheng's study had further revealed that similarity exists as a dual concept concerning the distance measure. In this article we shall find the Hausdorff distance to find the critical position, mid-position, and normal position for the patents based on neutrosophic membership value, non-membership value, and indeterminacy. There are many types of COVID-19 patents here some spatial types have been discussed for defining the different membership criterion functions. Many researcher worked on COVID-19 disease [17-20] and decision making model based on different problems [21-24]. Many researcher work on data mining in different situation, like Olson and Shi [25] Introduction to business data mining, Shi et al. [26] Optimization based data mining: theory and applications, Gamelin et al. [27] Effect of high intensity intermittent training on heart rate variability in prepubescent children. Slifka [28] introduced "Clinical implications of deregulated cytokine production" Brown and Aaron [29] worked on "The politics of nature" Smith et al. [30] worked on "Future of health insurance" neutrosophic set and its application [31, 32].

Research of COVID-19, is based on data from a symptom tracker app, discovered that there are six different "types" of the condition involving various groups of symptoms. The data could open new possibilities for how the doctors can properly treat individual cases and predict the level of clinic care they would require. Researchers from King's College London studied data from approximately 1600 U.K. and U.S. patients who regularly logged their symptoms in the COVID Symptom Tracker App during March and April 2020.Typically, doctors will examine for key symptoms such as cough, fever, and loss of the sense of smell to detect COVID-19. The study, which has not been peer-reviewed, says the six different "types" of COVID-19 can vary by severity and come with their own set of symptoms. 
The six clusters of symptoms outlined in the study are:

Flu-like with no fever: Headache, loss of smell, muscle pains, cough, sore throat, chest pain, no fever.

Flu-like with fever: Headache, loss of smell, cough, sore throat, hoarseness, fever, loss of appetite.

Gastrointestinal: Headache, loss of smell, loss of appetite, diarrhea, sore throat, chest pain, no cough.

Severe level one, fatigue: Headache, loss of smell, cough, fever, hoarseness, chest pain, fatigue.

Severe level two, confusion: Headache, loss of smell, loss of appetite, cough, fever, hoarseness, sore throat, chest pain, fatigue, confusion, muscle pain.

Severe level three, abdominal and respiratory: Headache, loss of smell, loss of appetite, cough, fever, hoarseness, sore throat, chest pain, fatigue, confusion, muscle pain, shortness of breath, diarrhea, abdominal pain.

The first level, "flu-like with no fever," is associated with headaches, loss of smell, muscle pains, cough, sore throat, and chest pain. Patients at this level have a $1.5 \%$ chance of needing breathing support such as oxygen or a ventilator.

The second type, "flu-like with fever," includes symptoms like loss of appetite, headache, loss of smell, cough, sore throat, hoarseness, and fever. Researchers say about $4.4 \%$ of patients at this level needed breathing support.

Patients with the third type, simply described as "gastrointestinal," do not have a cough as part of their illness. Instead, they experience headaches, diarrhea, loss of smell, loss of appetite, sore throat, and chest pain, and about 3.3\% needed breathing support.

Lahita referred to the following three clusters of COVID-19 as the "really severe types."

In type four, or "severe level one," patients experience fatigue along with headache, loss of smell, cough, fever, hoarseness, and chest pain. Patients at this level needed breathing support at a rate of $8.6 \%$.

Type five, "severe level two," includes the symptoms of type four along with loss of appetite, sore throat, and muscle pain, and is mainly distinguished by confusion.

"That means you don't know where you are or where you live, whether you are in or out of the hospital, who your relatives are," Lahita explained. "That is very scary." Almost $10 \%$ of patients at that level need breathing support.

How the coronavirus almost killed a healthy woman with "no normal symptoms".

The most severe type of COVID-19 is referred to as "severe level three, abdominal and respiratory," and has all the above symptoms along with abdominal pain, shortness of breath, and diarrhea. Nearly $20 \%$ of these patients need breathing support.

Lahita said "Those are the severe level threes who wind up on a ventilator, and then it is touch-and-go as to whether they survive the infection entirely".

The U.K. researchers have observed that only $16 \%$ of patients with type one COVID19 required hospitalization, compared with nearly half of the patients with type six.

Patients in the severe clusters also tend to be older or with pre-existing conditions and weakened immune systems, compared to those in the first three. 
We hope that it would help to predict the types of care patients with COVID-19 might need, and give doctors the ability to predict which patients would fall into which category.

Data Science and related technologies are critical in the fight against pandemics, such as the 2003 severe acute respiratory syndrome coronavirus (SARS-CoV), COVID-19, to enable governments and health managements figure out the best preparation and response. Big data, data mining, machine learning, and a variety of other technologies can be used to swiftly and effectively evaluate data in order to track and regulate COVID-19's spread over the world [17-20]. Big data is currently a hot topic among researchers, engineers, health-care executives, and administrators [26]. Several academics have made extensive use of data mining techniques to uncover hidden information in large datasets [19-21]. The results obtained from the study by Liu et al. [19] can be linked to India and other countries, which will be valuable in understanding the virus transmission patterns among the population of different age-groups.

In this paper we have prepared a C-programming by which is can compare suspected patents with the COVID-19 patents a prediction based on the expert data.

\section{Preliminaries}

In this section we procure some definitions and examples which will be used in rest of the article.

Definition 2.1 [6, 7] Let $U$ be an initial universe and $E$ be a set of parameters. Let $P(U)$ denotes the power set of $\mathrm{U}$ and $A \subseteq E$. Then the pair $(F, A)$ is called a soft set over $U$, where $F$ is a mapping given by $F: A \rightarrow P(U)$.

Definition 2.2 [32] A neutrosophic set $A$ on the universe of discourse $X$ is defined as $A=\left\{\left(\mathrm{x},\left(T_{A}(x), I_{A}(x), F_{A}(x)\right), x \widehat{I} X\right\} \text { where } T, I, F: X \rightarrow\right]^{-} 0,1^{+}\left[\right.$and $^{-} 0 \leq$ $T_{A}(x)+I_{\mathrm{A}}(x)+F_{A}(x) \leq 3^{+}$. From philosophical point of view, the neutrosophic set takes the value from real standard or non-standard subsets of $]^{-} 0,+1$ [But in real life application in scientific and engineering problems it is difficult to use neutrosophic set with value from real standard or non-standard subset of $]^{-} 0,1^{+}[$.

Hence we consider the neutrosophic set which takes the value from the subset of $[0,1]$ that is

$$
{ }^{-} 0 \leq T_{A}(x)+I_{A}(x)+F_{A}(x) \leq 3^{+},
$$

where $T_{A}(x)$ is called truth-membership function, $I_{A}(x)$ is called an indeterminacymembership function and $F_{A}(x)$ is called a falsity membership function.

Definition 2.3 [6] Let $U$ be the universal set and $E$ be the set of parameters. Let $A \subseteq E$ and $P(U)$ be the set of all neutrosophic sets of $U$. Then the collection $(F, A)$ is called neutrosophic soft set over $U$, where $\mathrm{F}$ is a mapping given by $F: A \rightarrow \mathrm{P}(U)$.

Definition 2.4 [32] Let $U$ is a space of points (objects), with a generic element in $U$. A single value neutrosophic set (SVN-set) $A$ in $U$ is characterized by truth membership function $T_{A}$, a indeterminacy-membership function $I_{A}$ and a falsity-membership 
function $F_{A}$. For each point $u \in U ; T_{A}, I_{A}$ and $F_{A} \in[0,1]$. Thus a $S V N$-set $A$ over $U$ is represented as $A=\left\{\left(T_{A}(u), I_{A}(u), F_{A}(u)\right): u \in \mathrm{U}\right\}$ Where ${ }^{-} 0 \leq T_{A}(u)+I_{A}(u)+$ $F_{A}(u) \leq 3^{+}$and $\left(T_{A}(u), I_{A}(u), F_{A}(u)\right)$ is called single value neutrosophic number for all $u$.

Definition 2.5 [7] Let $U$ be an initial universal set, $E$ be a set of parameters and $A$ $\subseteq E$. Let $\operatorname{SVNS}(U)$ denotes the set of all interval value neutrosophic subsets of $U$. The collection $(F, A)$ is termed to be the interval valued neutrosophic soft set over $U$, where $F$ is a mapping given by $F: A \rightarrow \operatorname{SVNS}(U)$.

\section{Similarity Measure Between Two IVNS Sets Based on Distances}

In this section we define hamming and Euclidean distances between two single valued neutrosophic sets and proposed similarity measures based on these distances.

COVID-19 disease is a severe issue that has affected many people around the world. The following are some of the state's most common symptoms.

A. Fever is a temperature measurement in degrees Celsius. Coughing that is dry, C. Exhaustion, E. Loss of taste or smell, D. Headache F. shortness of breath or difficulty breathing.

We create a neutrosophic soft set based on those symptoms, and by establishing a distance function, we create a C-programming that allows us to determine whether a patent Covid is positive or not. In this case, we'll use conventional Covid-19 patent data to calculate the distance between two regular patents; if the distance is zero, the patent is considered Covid positive. If the distance is great, we can assume the patents will have other issues.

If a patent has other issues, the percentage can be calculated using the neutrosophic distance.

Definition 3.1 Let $\mathrm{U}=\left\{x_{1}, x_{2}, x_{3}, \ldots \ldots, x_{n}\right\}$ be an initi al universe and $\mathrm{E}=\left\{\mathrm{e}_{1}\right.$, $\left.\mathrm{e}_{2}, \mathrm{e}_{3}, \ldots \ldots, \mathrm{e}_{m}\right\}$ be a set of parameters. Let $\operatorname{SVNS}(U)$ denotes the set of all single valued neutrosophic subsets of $U$. Also let $(A, E)$ and $(B, E)$ be two single valued neutrosophic soft sets over $U$, where $A$ and $B$ are mappings given by $A, B: E \rightarrow$ $\operatorname{SVNS}(U)$. Define the following distances between $(A, E)$ and $(B, E)$ as follows:

1. Hamming Distance

$$
\begin{aligned}
H D(A, E)= & \sum_{j=1}^{m} \sum_{i=1}^{n}\left(\left|T_{A}\left(x_{j}\right)\left(e_{i}\right)-T_{E}\left(x_{j}\right)\left(e_{i}\right)\right|+\left|I_{A}\left(x_{j}\right)\left(e_{i}\right)-I_{E}\left(x_{j}\right)\left(e_{i}\right)\right|\right. \\
& \left.+\left|F_{A}\left(x_{j}\right)\left(e_{i}\right)-F_{E}\left(x_{j}\right)\left(e_{i}\right)\right|\right)
\end{aligned}
$$

2. Normalized Hamming distance

$$
N H D(A, E)=\frac{1}{3 m n} \sum_{j=1}^{m} \sum_{i=1}^{n}\left(\left|T_{A}\left(x_{j}\right)\left(e_{i}\right)-T_{E}\left(x_{j}\right)\left(e_{i}\right)\right|+\left|I_{A}\left(x_{j}\right)\left(e_{i}\right)-I_{E}\left(x_{j}\right)\left(e_{i}\right)\right|\right.
$$




$$
\left.+\left|F_{A}\left(x_{j}\right)\left(e_{i}\right)-F_{E}\left(x_{j}\right)\left(e_{i}\right)\right|\right)
$$

3. Euclidean distance

$$
\begin{aligned}
E D(A, E)= & \sqrt{\sum_{j=1}^{m} \sum_{i=1}^{n}\left(\left|T_{A}\left(x_{j}\right)\left(e_{i}\right)-T_{E}\left(x_{j}\right)\left(e_{i}\right)\right|^{2}+\left|I_{A}\left(x_{j}\right)\left(e_{i}\right)-I_{E}\left(x_{j}\right)\left(e_{i}\right)\right|^{2}\right.} \\
& \left.+\left|F_{A}\left(x_{j}\right)\left(e_{i}\right)-F_{E}\left(x_{j}\right)\left(e_{i}\right)\right|^{2}\right)
\end{aligned}
$$

4. Normalized Euclidean distance

$$
\begin{aligned}
N E D(A, E)= & \sqrt{\frac{1}{3 n m} \sum_{j=1}^{m} \sum_{i=1}^{n}\left(\left|T_{A}\left(x_{j}\right)\left(e_{i}\right)-T_{E}\left(x_{j}\right)\left(e_{i}\right)\right|^{2}+\left|I_{A}\left(x_{j}\right)\left(e_{i}\right)-I_{E}\left(x_{j}\right)\left(e_{i}\right)\right|^{2}\right.} \\
& \left.+\left|F_{A}\left(x_{j}\right)\left(e_{i}\right)-F_{E}\left(x_{j}\right)\left(e_{i}\right)\right|^{2}\right)
\end{aligned}
$$

5. Neutrosophic Distance

$$
\begin{aligned}
N D(A, B)= & \left(\sum _ { j = 1 } ^ { m } \sum _ { i = 1 } ^ { n } \left(\left|T_{A}\left(x_{j}\right)\left(e_{i}\right)-T_{B}\left(x_{j}\right)\left(e_{i}\right)\right|,\right.\right. \\
& \sum_{j=1}^{m} \sum_{i=1}^{n}\left(\left|I_{A}\left(x_{j}\right)\left(e_{i}\right)-I_{B}\left(x_{j}\right)\left(e_{i}\right)\right|,\right. \\
& \left.\sum_{j=1}^{m} \sum_{i=1}^{n}\left|F_{A}\left(x_{j}\right)\left(e_{i}\right)-F_{B}\left(x_{j}\right)\left(e_{i}\right)\right|\right) \\
= & \left(\bar{T}\left(e_{i}\right), \bar{I}\left(e_{i}\right), \bar{F}\left(e_{i}\right)\right)=(\mathrm{m}, \mathrm{h}, \mathrm{g}),
\end{aligned}
$$

where, $\mu$ is the true membership value for covid $-19 \eta$ is the indeterminacy element. 6. Normalized Neutrosophic Distance

$$
\begin{aligned}
D_{\mathrm{N}}(A, B)= & \left(\frac { 1 } { n m } \sum _ { j = 1 } ^ { m } \sum _ { i = 1 } ^ { n } \left(\left|T_{A}\left(x_{j}\right)\left(e_{i}\right)-T_{B}\left(x_{j}\right)\left(e_{i}\right)\right|,\right.\right. \\
& \frac{1}{n m} \sum_{j=1}^{m} \sum_{i=1}^{n}\left(\left|I_{A}\left(x_{j}\right)\left(e_{i}\right)-I_{B}\left(x_{j}\right)\left(e_{i}\right)\right|,\right. \\
& \left.\frac{1}{n m} \sum_{j=1}^{m} \sum_{i=1}^{n}\left|F_{A}\left(x_{j}\right)\left(e_{i}\right)-F_{B}\left(x_{j}\right)\left(e_{i}\right)\right|\right)=\left(\bar{T}\left(x_{i}\right), \bar{I}\left(x_{i}\right), \bar{F}\left(x_{i}\right)\right) \\
<\mathrm{T}, \mathrm{I}, \mathrm{F}>= & \left\langle\frac{\bar{T}\left(x_{i}\right)}{\bar{T}\left(x_{i}\right)+\bar{I}\left(x_{i}\right)+\bar{F}\left(x_{i}\right.}, \frac{\bar{I}\left(x_{i}\right)}{\bar{T}\left(x_{i}\right)+\bar{I}\left(x_{i}\right)+\bar{F}\left(x_{i}\right)}, \frac{\bar{T}\left(x_{i}\right)+\bar{I}\left(x_{i}\right)+\bar{F}\left(x_{i}\right.}{\bar{T}}\right\rangle
\end{aligned}
$$

The percentage of possibility for Covid-19 positive is $\mathrm{T}^{*} 100 \%$, 
The percentage of doubt for Covid-19 positive is I*100\%,.

The percentage of possibility for Covid-19 negative is $\mathrm{F}^{*} 100 \%$,

In case of neutrosophic distance if $\mathrm{T}^{*} 100 \%, \mathrm{Z} \mathrm{I}^{*} 100 \%, .+\mathrm{F}^{*} 100 \%$, then the decision will be in favor of true membership value.

We have defined three types of distance function for the finding the distance between two patents where one patents have covid-19 and other one will measure by the distance function and what the percentage of covid positive occur.

If the distance lies in the interval $[0,0.4]$ then we consider both has the similar condition if the distance lies $(0.4,0.55)$ then cannot decided it must take under observation and if distance lies in $[0.55,1]$ then we consider the patents may have opposite decision.

\section{Application on COVID-19}

First we construct a decision making method based on similarity measure of two neutrosophic sets. It is a medical diagnosis problem on COVID-19.

Similarity measure of two neutrosophic soft sets based on Normalize Hamming distance, and Normalized Euclidian distance can be applied to estimate the possibility that an ill person having certain symptoms. He /She is suffering from COVID-19 or not. We construct a neutrosophic soft set for the disease and another neutrosophic soft set for ill person. Then we find the similarity measure of these two neutrosophic soft sets. If the similarity measure is less than or equal to 0.4 (which can be fixed by a medical expert person) then we conclude that the person is possibly suffering from COVID-19. If the similarity measure is greater than 0.4 to 0.55 then the person is not possibly suffering from the disease COVID-19 but need observation. similarity measure is greater than or equal to 0.55 then the patents are free from COVID-19.

The steps of this algorithm are:

Step 1 A neutrosophic soft set element is Constructed for disease over the universe $U$. It is based on medical expert.

Step 2 A neutrosophic soft set $(G, B)$ is Constructed over the universe $U$ for a patient.

Step 3 Calculate the Hamming distance between $(\mathrm{F}, E)$ and $(G, E)$.

Step 4 Calculate similarity measure of $(F, E)$ and $(G, E)$.

Step 5 Estimate result by using the similarity.

Example 1 Here we are providing an example of a decision-making method in a single-valued neutrosophic soft set setting using a similarity measure.

To handle that pandemic situation, select an ideal case (a patient who was suffering from COVID-19). By calculation of similarity measure of any suspected patient with an ideal case to the diagnosis of the COVID-19. Through this technique, doctors can take fast a decision to diagnosis the disease and create a helping environment in medical diagnosis.

In this case, let us consider a patient who was suffering from COVID-19. Using the COVID-19 patient data we will decide for a set of elements of new patients. $\mathrm{P}_{1}, \mathrm{P}_{2}$, $\mathrm{P}_{3}$ and universal set $U$ of Experts. Let $U=\left\{x_{1}, x_{2}, x_{3}, x_{4}\right\}$ be the universal set and $E$ 
$=\left\{e_{1}, e_{2}, e_{3}, e_{4}, e_{5}, e_{6}\right\}$ be the set of parameters. Let $(A, E),\left(P_{1}, E\right),\left(A, P_{1}\right)$ and $(A$, $\left.P_{3}\right)$ are four neutrosophic soft sets over $U$. Here $A$ is the known person we have to find out the distance of $P_{i}$ from $A$. The tabular representations of $A$ and $\mathrm{P}_{\mathrm{i}}$ 'sare as follows:

\begin{tabular}{lllllll}
$(A, E)$ & $e_{1}$ & $e_{2}$ & $e_{3}$ & $e_{4}$ & $e_{5}$ & $e_{6}$ \\
\hline$x_{1}$ & $<1,0,0>$ & $<1,0,0>$ & $<1,0,0>$ & $<1,0,0>$ & $<1,0,0>$ & $<1,0,0>$ \\
$x_{2}$ & $<1,0,0>$ & $<1,0,0>$ & $<1,0,0>$ & $<1,0,0>$ & $<1,0,0>$ & $<1,0,0>$ \\
$x_{3}$ & $<1,0,0>$ & $<1,0,0>$ & $<1,0,0>$ & $<1,0,0>$ & $<1,0,0>$ & $<1,0,0>$ \\
$x_{4}$ & $<1,0,0>$ & $<1,0,0>$ & $<1,0,0>$ & $<1,0,0>$ & $<1,0,0>$ & $<1,0,0>$ \\
\hline
\end{tabular}

\begin{tabular}{lcccccc}
\hline$\left.P_{1}, E\right)$ & $e_{1}$ & $e_{2}$ & $e_{3}$ & $e_{4}$ & $e_{5}$ & $e_{6}$ \\
\hline$x_{1}$ & $<0.1,0.2$, & $<0.2,0.3$, & $<0.1,0.1$, & $<0.2,0.3$, & $<0.21,0.2$, & $<0.1,0.3$, \\
& $0.7>$ & $0.5>$ & $0.9>$ & $0.5>$ & $0.9>$ & $0.9>$ \\
$x_{2}$ & $<0.1,0.6$, & $<0.2,0.5$, & $<0.1,0.2$, & $<0.1,0.5$, & $<0.3,0.2$, & $<0.1,0.2$, \\
& $0.6>$ & $0.7>$ & $0.8>$ & $0.4>$ & $0.5>$ & $0.6>$ \\
$x_{3}$ & $<0.3,0.2$, & $<0.3,0.4$, & $<0.1,0.2$, & $<0.2,0.3$, & $<0.2,0.6$, & $<0.0,0.6$, \\
& $0.6>$ & $0.6>$ & $0.7>$ & $0.5>$ & $0.9>$ & $0.9>$ \\
$x_{4}$ & $<0.1,0.1$, & $<0.2,0.3$, & $<0,0.1$, & $<0.1,0.2$, & $<0.2,0.5$, & $<0.4,0.1$, \\
& $0.9>$ & $0.5>$ & $0.9>$ & $0.7>$ & $0.3>$ & $0.5>$ \\
\hline
\end{tabular}

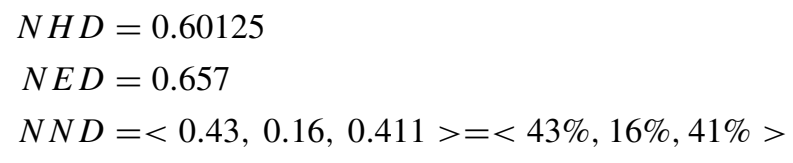

\begin{tabular}{lcccccc}
\hline$\left.P_{2}, E\right)$ & $e_{1}$ & $e_{2}$ & $e_{3}$ & $e_{4}$ & $e_{5}$ & $e_{6}$ \\
\hline$x_{1}$ & $<0.5,0.4$, & $<0.4,0.4$, & $<0.5,0.5$, & $<0.1,0.4$, & $<0.7,0.3$, & $<0.8,0.2$, \\
& $0.2>$ & $0.3>$ & $0>$ & $0.5>$ & $0>$ & $0>$ \\
$x_{2}$ & $<0.4,0.5$, & $<0.3,0.4$, & $<0.5,0.2$, & $0.2,0.3,0.4$ & $<0.8,0$, & $<0.7,0$, \\
& $0.1>$ & $0.3>$ & $0.3>$ & $>$ & $0.2>$ & $0.3>$ \\
$x_{3}$ & $<0.6,0.4$, & $<0.5,0.5$, & $<0.4,0.2$, & $<0.1,0.2$, & $<0.5,0.2$, & $<0.6,0.1$, \\
& $0>$ & $0>$ & $0.4>$ & $0.7>$ & $0.3>$ & $0.3>$ \\
$x_{4}$ & $<0.5,0.2$, & $<0.2,0.3$, & $<0.4,0.3$, & $<0.3,0.2$, & $<0.6,0.2$, & $<0.8,0.1$, \\
& $0.3>$ & $0.5>$ & $0.3>$ & $0.5>$ & $0.2>$ & $0.1>$ \\
\hline
\end{tabular}




$$
\begin{aligned}
& N H D=0.351388 ; N E D=0.36836 \\
& N N D=<0.5,0.26,0.24>=<50 \%, 26 \%, 24 \%>
\end{aligned}
$$

\begin{tabular}{lcccccc}
\hline$\left(P_{3}, E\right)$ & $e_{1}$ & $e_{2}$ & $e_{3}$ & $e_{4}$ & $e_{5}$ & $e_{6}$ \\
\hline$x_{1}$ & $<0.8,0$, & $<0.7,0.1$, & $<0.8,0.1$, & $<0.7,0.2$, & $<0.6,0.2$, & $<0.7,0.2$, \\
& $0.2>$ & $0.2>$ & $0.1>$ & $0.1>$ & $0.2>$ & $0.1>$ \\
$x_{2}$ & $<0.7,0.3$, & $<0.8,0$, & $<0.9,0.1$, & $<0.8,0$, & $<0.7,0.2$, & $<0.6,0.1$, \\
& $0>$ & $0.2>$ & $0>$ & $0.2>$ & $0.1>$ & $0.3>$ \\
$x_{3}$ & $<0.8,0.2$, & $<0.7,0.2$, & $<0.6,0.1$, & $<0.7,0.1$, & $<0.6,0.4$, & $<0.7,0.1$, \\
& $0>$ & $0.1>$ & $0.3>$ & $0.2>$ & $0>$ & $0.2>$ \\
$x_{4}$ & $<0.7,0.1$, & $<0.6,0.2$, & $<0.7,0.1$, & $<0.6,0.2$, & $<0.6,0.2$, & $<0.6,0.3$, \\
& $0.2>$ & $0.2>$ & $0.2>$ & $0.2>$ & $0.2>$ & $0.1>$ \\
\hline
\end{tabular}

$$
\begin{aligned}
& N H D=0.2027777 ; N E D=0.2327258 \\
& N N D=<0.5,0.25,0.25>=<50 \%, 25 \%, 25 \%>
\end{aligned}
$$

\subsection{Calculation of Normalize Hamming Distances}

Now by Definition 3.1 the Normalized Hamming distance between $A$ and $P_{1}, P_{2}, P_{3}$ are given by

$$
N H D\left(A, P_{1}\right)=0.60125, N H D\left(A, P_{2}\right)=0.351388, N H D\left(A, P_{3}\right)=0.202777
$$

According to the Definition 3.1 the decision by the above distance the patents $P_{1}$, $P_{2}, P_{3}$ are as follows

$$
\begin{aligned}
& P 1-\text { ve } \\
& P 2+\text { ve } \\
& P 3+\text { ve }
\end{aligned}
$$

\subsection{Calculation Normalize Euclidean Distances}

Now by Definition 3.1 Normalize Euclidean distancesbetween $A$ and $P 1, P 2, P 3$ are given by,

$\operatorname{NED}\left(A, P_{1}\right)=0.657, \operatorname{NED}\left(A, P_{2}\right)=0.368, \operatorname{NED}\left(A, P_{3}\right)=0.2327$. 
According to the Definition 3.1 the decision about the patents $P_{1}, P_{2}, P_{3}$ are as follows

$$
\begin{aligned}
& P 1-\text { ve } \\
& P 2+\text { ve } \\
& P 3+\text { ve }
\end{aligned}
$$

\subsection{Calculation of Normalize Neutrosophic Distances}

Now by Definition 3.1 Normalize Neutrosophic distancesbetween $A$ and $P 1, P 2, P 3$ are given by,

$$
\begin{aligned}
& N N D\left(A, P_{1}\right)=<0.43,0.16,0.41>=<43 \%, 16 \%, 41 \%> \\
& N N D\left(A, P_{2}\right)=<0.5,0.26,0.24>=<50 \%, 26 \%, 24 \%> \\
& N N D\left(A, P_{3}\right)=<0.5,0.25,0.25>=<50 \%, 25 \%, 25 \%>
\end{aligned}
$$

According to the Definition 3.1 the decision about the patents $P_{1}, P_{2}, P_{3}$ are as follows

$$
\begin{aligned}
& P 1-\text { ve } \\
& P 2+\text { ve } \\
& P 3+\text { ve }
\end{aligned}
$$

After that calculation the results are as follows:

Here we consider patents A as a Covid-19.

\subsection{Patients Results}

$$
\begin{aligned}
& \text { P1 - ve } \\
& \text { P2 + ve } \\
& \text { P3 + ve }
\end{aligned}
$$

\section{Conclusion}

In this paper we established a c-programming for finding the distances between two single valued neutrosophic soft sets. We use different distances function for similarity measure between two single valued neutrosophic soft sets. A programming is developed using for decision making problem. An example is given to demonstrate the possible application of proposed C-program in COVID-19 patients. The article usable 
for other decision making problems also, which is quite relevant to neutrosophic soft set.

Note The work is executed by the C-programming. The programming is given in the Appendix below.

Acknowledgements The work of the first author is financially supported by the Council of Scientific and Industrial Research, India vide Ref. No: 17/06/2018(i) EU-V and File No. 09/714(0022)/2019-EMR-I. The authors thank the reviewers for their comments, those improved the presentation of the article.

Author Contributions All author have equal contributions.

Funding Not applicable.

Data Availability Not applicable.

Code Availability Not applicable.

\section{Declarations}

Conflict of interest Authors declare that they have no conflict of interest. 


\title{
Appendix
}

\author{
\#include <stdio.h> \\ $\#$ \#include<conio.h> \\ \#include<math.h> \\ void main() \{ \\ int $n, k, i, j, l, m$;
}

float x[10][10][10][3],HD[10],ED[10],NHD[10],NED[10], T[10],F[10],I[10], S[10];

$\operatorname{clrscr}()$;

printf("Enter the number of syntoms n");

scanf("\%d",\&n);

printf("Enter the number of patents k");

scanf("\%d",\&k);

printf("Enter the number of eaxparts m");

$\operatorname{scanf}(" \% d ", \& m)$;

printf("Enter the Neutrosophic Data of all patents includind ill patents in 1st position");

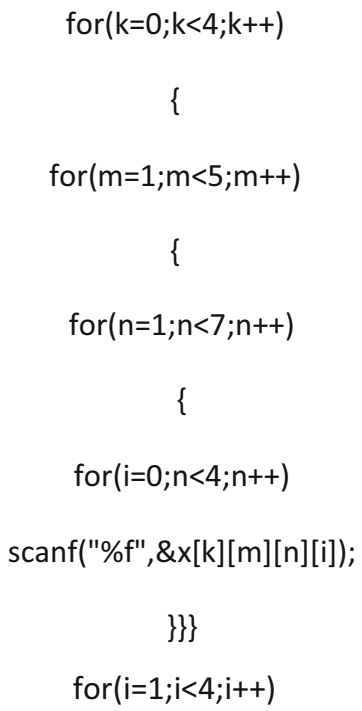




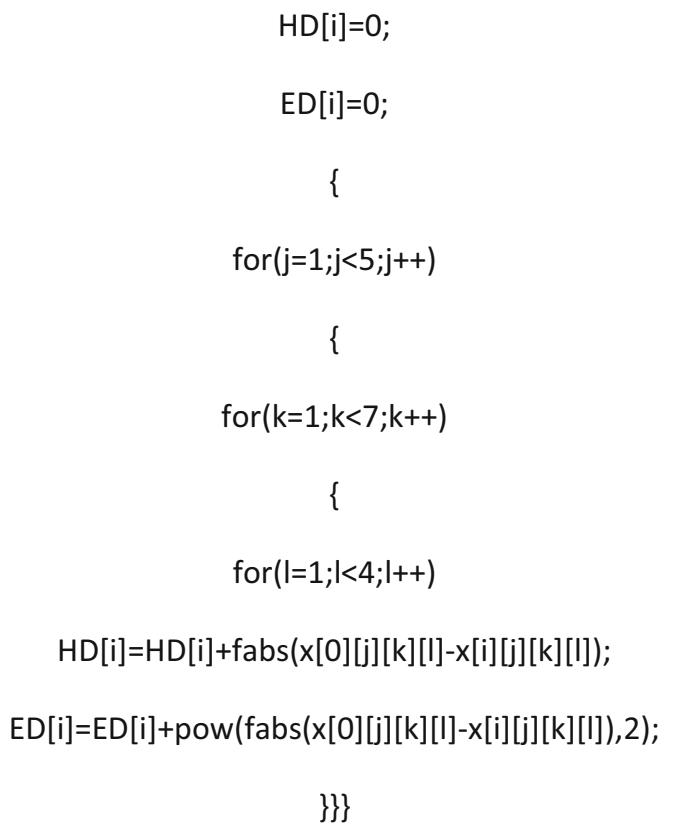

printf("the normalized Hamming distance and Ecludian distance are following: \n ");

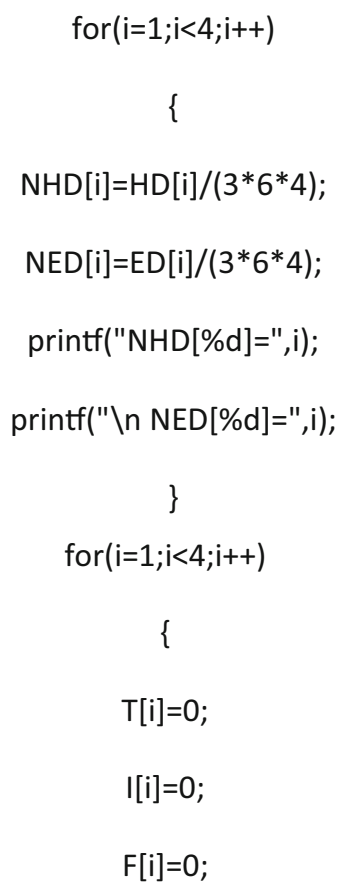




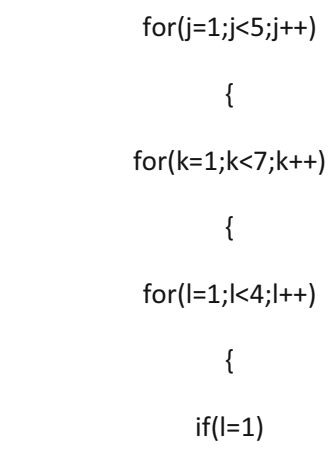

$T[i]=T[i]+f a b s(x[0][j][k][l]-x[i][j][k][l])$; else if(l=2)

$I[i]=I[i]+f a b s(x[0][j][k][l]-x[i][j][k][I])$;

else

I[i]=I[i]+fabs $(x[0][j][k][I]-x[i][j][k][I])$;

\}\}\}\}

printf("The normalized Neutrosophic distance is as follows $\backslash n$ ");

$$
\begin{gathered}
\text { for }(i=1 ; i<4 ; i++) \\
\{ \\
S[i]=T[i]+I[i]+F[i] ; \\
T[i]=(T[i] /(6 * 4 * S[i])) * 100 ; \\
I[i]=(I[i] /(6 * 4 * S[i])) * 100 ; \\
F[i]=(F[i] /(6 * 4 * S[i])) * 100 ;
\end{gathered}
$$

printf("The patents \%d has \%f percentage probability to have Covid positive",i,T[i]); printf("The patents \%d has \%f percentage Doubt to have Covid positive",i,I[i]); printf("The patents \%d has \%f percentage Probability to do not have Covid positive",i,[i]);

\}

printf("From the Above conclusion the Results of the given patents can be analysis and any patents can take the decision on the basis of given data $(n$ ")

$\operatorname{getch}() ;\}$ 


\section{References}

1. Atanassov K (1986) Intuitionistic fuzzy sets. Fuzzy Sets Syst 20:87-96

2. Atanassov K, Gargov G (1989) Interval valued intuitionistic fuzzy sets. Fuzzy Sets Syst 31:343-349

3. Park JH, Lim KM, Park JS, Kwun YC (2008) Distances between interval-valued intuitionistic fuzzy sets. J Phys Conf Ser 96:012089

4. Maji PK, Biswas R, Roy AR (2004) Intuitionistic fuzzy soft sets. J Fuzzy Math 12(3):669-683

5. Wang PZ (1983) Fuzzy sets and its applications, Shanghai Science and Technology Press. Shanghai 1983 in Chinese

6. Molodtsov D (1999) Soft set theory-first results. Comput Math Appl 37:19-31

7. Cagman N, Deli I (2013) Similarity measure of intuitionistic fuzzy soft sets and their decision making, arXiv: 1301.0456vI [math.LO] 3jan 2013

8. Chen SM (1997) Similarity measures between vague sets and between elements. IEEE Trans Syst Man Cybern Part B 27(1):153-168

9. Hu K, Li J (2013) The entropy and similarity measure of interval valued intuitionistic fuzzy sets and their relationship. Int J Fuzzy Syst 15(3):279-288

10. Majumdar P, Samanta SK (2008) Similarity measure of soft sets. New Math Nat Comput 4(1):1-12

11. Majumdar P, Samanta SK (2011) On similarity measures of fuzzy soft sets. Int J Adv Soft Comput Appl 3(2):1-8

12. Majumdar P, Samanta SK (2010) On distance based similarity measure between intuitionistic fuzzy soft sets. Anusandhan 12(22):41-50

13. Min WK (2012) Similarity in soft set theory. Appl Math Lett 25:310-314

14. Mukherjee A, Sarkar S (2014) Similarity measures of interval-valued fuzzy soft sets. Ann Fuzzy Math Inform 8(9):447-460

15. Mukherjee A, Sarkar S (2014) Similarity measures for interval-valued intuitionistic fuzzy soft sets and its application in medical diagnosis problem. New Trends Math Sci 2(3):159-165

16. Xuecheng L (1992) Entropy distance and similarity measure of fuzzy sets and their relations. Fuzzy Sets Syst 52(3):305-318

17. Kumar S (2020) Monitoring novel corona virus (COVID-19) infections in India by cluster analysis. Ann Data Sci 7:417-425

18. Li J, Guo K, Herrera Viedma E, Lee H, Liu J, Zhong Z, Gomes L, Filip FG, Fang SC, Özdemir MS, Liu XH, Lu G, Sh Y (2020) Culture vs Policy: more global collaboration to effectively combat COVID-19. Innovation. https://doi.org/10.1016/j.xinn.2020.100023

19. Liu Y, Gu Z, Xia S, Shi B, Zhou X, Shi Y, Liu J (2020) What are the underlying transmission patterns of COVID-19 outbreak? An age-specific social contact characterization. EClincialMedicine 22:100354-100361

20. Majumder P, Das S, Das R, Tripathy BC (2021) Identification of the most significant risk factor of COVID-19 in economy using cosine similarity measure under SVPNS-environment. Neutrosophic Sets Syst 46:112-127. https://doi.org/10.5281/zenodo.5553497

21. Mukherjee A, Das R (2020) Neutrosophic bipolar vague soft set and its application to decision making problems. Neutrosophic Sets Syst 32:410-424

22. Tien JM (2017) Internet of things, real-time decision making, and artificial intelligence. Ann Data Sci 4(2):149-178

23. Das S, Das R, Tripathy BC (2020) Multi-criteria group decision making model using single-valued neutrosophic set. LogForum 16(3):421-429

24. Temesgen A, Gurmesa A, Getchew Y (2018) Joint modeling of Longitudinal CD4 count and time-todeath of HIV/TB Co-infected patients: a case of Jimma University Specialized Hospital. Ann Data Sci 5:659-678

25. Olson DL, Shi Y (2007) Introduction to business data mining. McGraw-Hill/Irwin, New York

26. Shi Y, Tian YJ, Kou G, Peng Y, Li JP (2011) Optimization based data mining: theory and applications. Springer, Berlin

27. Gamelin FX, Baquet G, Berthoin S, Thevenet D, Nourry C, Nottin S, Bosquet L (2009) Effect of high intensity intermittent training on heart rate variability in prepubescent children. Eur $\mathbf{J}$ Appl Physiol 105:731-738. https://doi.org/10.1007/s00421-008-0955-8

28. Slifka MK, Whitton JL (2000) Clinical implications of dysregulated cytokine production. J Mol Med. https://doi.org/10.1007/s001090000086 
29. Brown B, Aaron M (2001) The politics of nature. In: Smith J (ed) The rise of modern genomics, 3rd edn. Wiley, New York, pp 230-257

30. Smith J, Jones M Jr, Houghton L et al (1999) Future of health insurance. N Engl J Med 965:325-329

31. Das R, Smarandache F, Tripathy BC (2020) Neutrosophic fuzzy matrices and some algebraic operations. Neutrosophic Sets Syst 32:401-409

32. Smarandache F (1998) A unifying field in logics, neutrosophy: neutrosophic probability, set and logic. American Research Press, Rehoboth

Publisher's Note Springer Nature remains neutral with regard to jurisdictional claims in published maps and institutional affiliations. 